\title{
A TWO-WEIGHT ESTIMATE FOR A CLASS OF FRACTIONAL INTEGRAL OPERATORS WITH ROUGH KERNEL
}

\author{
YONG DING
}

Received 20 November 2004 and in revised form 16 June 2005

We prove that the operators in a class of rough fractional integral operators and the related maximal operators are bounded from $L^{p}\left(v^{p}\right)$ to $L^{q}\left(u^{q}\right)$ with weight pair $(u, v)$.

\section{Introduction and results}

Suppose that $\Omega \in L^{s}\left(S^{n-1}\right)(s \geq 1)$ is homogeneous of degree zero in $\mathbb{R}^{n}$ with zero mean value on $S^{n-1}$, then it is well known that the Calderón-Zygmund singular integral is defined by

$$
T_{\Omega} f(x)=p \cdot v \cdot \int_{\mathbb{R}^{n}} \frac{\Omega(x-y)}{|x-y|^{n}} f(y) d y .
$$

In 1967, Bajšanski and Coifman [1] first discussed the boundedness of operators on a class of singular integral operators $T_{\Omega}^{A}$ which are associated with the commutators of the Calderón-Zygmund singular integral $T_{\Omega}$. The operator $T_{\Omega}^{A}$ is defined by

$$
T_{\Omega}^{A} f(x)=p \cdot v \cdot \int_{\mathbb{R}^{n}} \frac{R_{m}(A, x, y)}{|x-y|^{n+m-1}} \Omega(x-y) f(y) d y,
$$

where $A(x)$ is defined on $\mathbb{R}^{n}$ and $R_{m}(A ; x, y)$ denotes the $m$ th remainder of the Taylor series of $A$ at $x$ about $y$. More precisely,

$$
R_{m}(A ; x, y)=A(x)-\sum_{|\gamma|<m} \frac{1}{\gamma !} D^{\gamma} A(y)(x-y)^{\gamma},
$$

where $D^{\gamma} A \in L^{r}\left(\mathbb{R}^{n}\right)(1<r \leq \infty)$ or $D^{\gamma} A \in \operatorname{BMO}\left(\mathbb{R}^{n}\right)$ for $|\gamma|=m-1(m \geq 1)$. Following [1], Cohen and Gosselin studied also the boundedness of $T_{\Omega}^{A}$ in $[2,3,4]$.

Notice that the fractional integral operator is closely related to the Calderón-Zygmund singular integral operator, which is defined by

$$
T_{\Omega, \alpha} f(x)=\int_{\mathbb{R}^{n}} \frac{\Omega(x-y)}{|x-y|^{n-\alpha}} f(y) d y
$$


for $0<\alpha<n$. (See $[5,7,9]$ for the boundedness of $T_{\Omega, \alpha}$.) Therefore, a natural and interesting problem is whether there are similar mapping properties if we replace the kernel function $\Omega(x)|x|^{-n}$ by $\Omega(x)|x|^{-(n-\alpha)}(0<\alpha<n)$ in definition (1.2). In this paper, we will consider the above problem. In a very simple way, which is altogether different from the method in $[3,4]$, we will obtain the weighted $\left(L^{p}, L^{q}\right)$ boundedness with the weight pair $(u, v)$ for the rough fractional integral operator $T_{\Omega, \alpha}^{A}$, which is defined by

$$
T_{\Omega, \alpha}^{A} f(x)=\int_{\mathbb{R}^{n}} \frac{R_{m}(A, x, y)}{|x-y|^{n-\alpha+m-1}} \Omega(x-y) f(y) d y,
$$

where $0<\alpha<n, \Omega \in L^{s}\left(S^{n-1}\right)(s>1)$ is homogeneous of degree zero in $\mathbb{R}^{n}$, and $R_{m}(A$; $x, y)$ is as in (1.3). As a corollary of the above result, in this paper, we show also that the same conclusion holds for the fractional maximal operator $M_{\Omega, \alpha}^{A}$, where

$$
M_{\Omega, \alpha}^{A} f(x)=\sup _{r>0} \frac{1}{r^{n-\alpha+m-1}} \int_{|x-y|<r}\left|R_{m}(A, x, y)\right||\Omega(x-y) f(y)| d y .
$$

Before stating our results, we give some definitions. In the following definitions, $p^{\prime}=$ $p /(p-1)$ and $Q$ denotes a cube in $\mathbb{R}^{n}$ with its sides parallel to the coordinate axes and the supremum is taken over all such cubes.

Definition $1.1\left(A_{p}(1<p<\infty)\right)$. A locally integrable nonnegative function $\omega(x)$ is said to belong to $A_{p}(1<p<\infty)$ if there is a constant $C>0$ such that

$$
\sup _{Q}\left(\frac{1}{|Q|} \int_{Q} \omega(x) d x\right)\left(\frac{1}{|Q|} \int_{Q} \omega(x)^{-1 /(p-1)} d x\right)^{p-1} \leq C .
$$

Definition $1.2(A(p, q)(1<p, q<\infty))$. A locally integrable nonnegative function $\omega(x)$ on $\mathbb{R}^{n}$ is said to belong to $A(p, q)(1<p, q<\infty)$ if there is a constant $C>0$ such that

$$
\sup _{Q}\left(\frac{1}{|Q|} \int_{Q} \omega(x)^{q} d x\right)^{1 / q}\left(\frac{1}{|Q|} \int_{Q} \omega(x)^{-p^{\prime}} d x\right)^{1 / p^{\prime}} \leq C<\infty .
$$

Definition $1.3\left(A_{p}^{*}(1<p<\infty)\right)$. A pair $(u(x), v(x))$ of nonnegative locally integrable functions is said to belong to $A_{p}^{*}(1<p<\infty)$ if there is a constant $C>0$ such that

$$
\sup _{Q}\left(\frac{1}{|Q|} \int_{Q} u(x) d x\right)\left(\frac{1}{|Q|} \int_{Q} v(x)^{-1 /(p-1)} d x\right)^{p-1} \leq C
$$

Definition $1.4\left(A^{*}(p, q)(1<p, q<\infty)\right)$. A pair $(u(x), v(x))$ of nonnegative locally integrable functions is said to belong to $A^{*}(p, q)(1<p, q<\infty)$ if there is a constant $C>0$ such that

$$
\sup _{Q}\left(\frac{1}{|Q|} \int_{Q} u(x)^{q} d x\right)^{1 / q}\left(\frac{1}{|Q|} \int_{Q} v(x)^{-p^{\prime}} d x\right)^{1 / p^{\prime}} \leq C<\infty
$$


Now we state the results obtained in this paper as follows.

Theorem 1.5. Let $\Omega \in L^{s}\left(S^{n-1}\right), 0<\alpha<n, 1<p<n / \alpha, D^{\gamma} A \in L^{r}\left(\mathbb{R}^{n}\right)(1<r \leq \infty),|\gamma|=$ $m-1,(m \geq 1)$, and $1 / q=1 / r+1 / p-\alpha / n$. Moreover, $1 / t=1 / r+1 / p$ and $1 / l=1 / p-\alpha / n$. If $s, l, t, p, q$ and $\omega$ satisfy one of the following conditions:

(a) $1 \leq s^{\prime}<t$ and $\left(u^{l s^{\prime} / q}, v^{l s^{\prime} / q}\right) \in A^{*}\left(t / s^{\prime}, q / s^{\prime}\right)$, and $u^{l s^{\prime} / q}, v^{l s^{\prime} / q} \in A\left(t / s^{\prime}, q / s^{\prime}\right)$;

(b) $s>l$ and $\left(v^{-t^{\prime} s^{\prime} / p^{\prime}}, u^{-t^{\prime} s^{\prime} / p^{\prime}}\right) \in A^{*}\left(l^{\prime} / s^{\prime}, p^{\prime} / s^{\prime}\right)$, and $v^{-t^{\prime} s^{\prime} / p^{\prime}}, u^{-t^{\prime} s^{\prime} / p^{\prime}} \in A\left(l^{\prime} / s^{\prime}, p^{\prime} / s^{\prime}\right)$, then there is a constant $C>0$, independent of $f$, such that

$$
\left(\int_{\mathbb{R}^{n}}\left|T_{\Omega, \alpha}^{A} f(x) u\right|^{q} d x\right)^{1 / q} \leq C \sum_{|\gamma|=m-1}\left\|D^{\gamma} A\right\|_{L^{r}}\left(\int_{\mathbb{R}^{n}}|f(x) v(x)|^{p} d x\right)^{1 / p}
$$

The proof of Theorem 1.5 depends on the following weighted $\left(L^{p}, L^{q}\right)$ boundedness and the weak boundedness of the rough fractional integral operator $T_{\Omega, \alpha}$.

Theorem 1.6 (see [6]). Suppose that $0<\alpha<n, 1<p<n / \alpha, 1 / q=1 / p-\alpha / n$, and $\Omega \in$ $L^{s}\left(S^{n-1}\right)$. If $s, p, q$, and $(u, v)$ satisfy one of the following conditions:

(a) $1 \leq s^{\prime}<p$ and $\left(u^{s^{\prime}}, v^{s^{\prime}}\right) \in A^{*}\left(p / s^{\prime}, q / s^{\prime}\right)$, and $u^{s^{\prime}}, v^{s^{\prime}} \in A\left(p / s^{\prime}, q / s^{\prime}\right)$;

(b) $s>q$ and $\left(v^{-s^{\prime}}, u^{-s^{\prime}}\right) \in A^{*}\left(q^{\prime} / s^{\prime}, p^{\prime} / s^{\prime}\right)$, and $v^{-s^{\prime}}, u^{-s^{\prime}} \in A\left(q^{\prime} / s^{\prime}, p^{\prime} / s^{\prime}\right)$,

then there is a constant $C>0$, independent of $f$, such that

$$
\left(\int_{\mathbb{R}^{n}}\left|T_{\Omega, \alpha} f(x) u\right|^{q} d x\right)^{1 / q} \leq C\left(\int_{\mathbb{R}^{n}}|f(x) v(x)|^{p} d x\right)^{1 / p} .
$$

\section{Some elementary conclusions}

We begin by giving some properties of the weight classes $A_{p}$ and $A_{p}^{*}$, which will be applied in the proof of theorem.

Some elementary properties of $A_{p}$ and $A_{p}^{*}(1<p<\infty)$ are as follows.

(i) $A_{p_{1}} \subset A_{p_{2}}$ if $1<p_{1} \leq p_{2}<\infty$.

(ii) If $(u, v) \in A_{p}^{*}$, then for any $0<\varepsilon<1,\left(u^{\varepsilon}, v^{\varepsilon}\right) \in A_{p}^{*}$.

(iii) If $(u, v) \in A_{p}^{*}$, then for any $r>p,(u, v) \in A_{r}^{*}$.

See $[8,6]$ for the proofs of (i)-(iii), respectively.

The relations between $A(p, q)$ and $A_{p}, A^{*}(p, q)$ and $A_{p}^{*}$ are explained as follows (see [6]). Suppose that $0<\alpha<n, 1<p<n / \alpha$ and $1 / q=1 / p-\alpha / n$, then

$$
\begin{gathered}
\omega(x) \in A(p, q) \Longleftrightarrow \omega(x)^{q} \in A_{q(n-\alpha) / n} \Longleftrightarrow \omega(x)^{-p^{\prime}} \in A_{1+p^{\prime} / q} ; \\
(u, v) \in A^{*}(p, q) \Longleftrightarrow\left(u^{q}, v^{q}\right) \in A_{q(n-\alpha) / n}^{*} \Longleftrightarrow\left(v^{-p^{\prime}}, u^{-p^{\prime}}\right) \in A_{1+p^{\prime} / q}^{*} .
\end{gathered}
$$

The proof of Theorem 1.5 is set up on the conclusions of the following two lemmas.

Lemma 2.1. Suppose that $0<\alpha<n, 1<p<n / \alpha, 1<r \leq \infty, 1 / q=1 / r+1 / p-\alpha / n$. Moreover, $1 / t=1 / r+1 / p, 1 / l=1 / p-\alpha / n$.

(a) If $1 \leq s^{\prime}<t$ and $\left(u^{l s^{\prime} / q}, v^{l s^{\prime}} / q\right) \in A^{*}\left(t / s^{\prime}, q / s^{\prime}\right)$, then $\left(u^{s^{\prime}}, v^{s^{\prime}}\right) \in A^{*}\left(p / s^{\prime}, l / s^{\prime}\right)$ and $\left(u^{s^{\prime}}\right.$, $\left.v^{s^{\prime}}\right) \in A^{*}\left(t / s^{\prime}, q / s^{\prime}\right)$.

(b) If $1 \leq s^{\prime}<t$ and $u^{l s^{\prime} / q}, v^{l s^{\prime} / q} \in A\left(t / s^{\prime}, q / s^{\prime}\right)$, then $u^{s^{\prime}}, v^{s^{\prime}} \in A\left(p / s^{\prime}, l / s^{\prime}\right)$ and $u^{s^{\prime}}, v^{s^{\prime}} \in$ $A\left(t / s^{\prime}, q / s^{\prime}\right)$. 
1838 Two-weight estimate for fractional integral operators

(c) If $s>l$ and $\left(v^{-s^{\prime} t^{\prime} / p^{\prime}}, u^{-s^{\prime} t^{\prime} / p^{\prime}}\right) \in A^{*}\left(l^{\prime} / s^{\prime}, p^{\prime} / s^{\prime}\right)$, then $\left(v^{-s^{\prime}}, u^{-s^{\prime}}\right) \in A^{*}\left(q^{\prime} / s^{\prime}, t^{\prime} / s^{\prime}\right)$ and $\left(v^{-s^{\prime}}, u^{-s^{\prime}}\right) \in A^{*}\left(l^{\prime} / s^{\prime}, p^{\prime} / s^{\prime}\right)$.

(d) If $s>l$ and $v^{-s^{\prime} t^{\prime} / p^{\prime}}, u^{-s^{\prime} t^{\prime} / p^{\prime}} \in A\left(l^{\prime} / s^{\prime}, p^{\prime} / s^{\prime}\right)$, then $v^{-s^{\prime}}, u^{-s^{\prime}} \in A\left(q^{\prime} / s^{\prime}, t^{\prime} / s^{\prime}\right)$ and $v^{-s^{\prime}}$, $u^{-s^{\prime}} \in A\left(l^{\prime} / s^{\prime}, p^{\prime} / s^{\prime}\right)$.

Proof. If $r=\infty$, then we have $t=p, l=q$. Obviously, the conclusions (a)-(d) in Lemma 2.1 hold. Hence, we need only to consider the case $1<r<\infty$. Below, we give the proof only for the conclusion (a), the proofs of conclusions (b) $-(\mathrm{d})$ are similar.

Since $s^{\prime}<t<p<n / \alpha$, we have $0<\alpha s^{\prime}<n, 1<t / s^{\prime}<n / \alpha s^{\prime}$, and $1 /\left(q / s^{\prime}\right)=1 /\left(t / s^{\prime}\right)-$ $\alpha s^{\prime} / n$. By $\left(u^{l s^{\prime} / q}, v^{l s^{\prime} / q}\right) \in A^{*}\left(t / s^{\prime}, q / s^{\prime}\right)$ and (2.2), we have

$$
\left(u^{l}, v^{l}\right) \in A_{q\left(n-\alpha s^{\prime}\right) / s^{\prime} n}^{*} .
$$

Noting that $q<l$, from $(2.3)$ and (iii), we get

$$
\left(u^{l}, v^{l}\right) \in A_{q\left(n-\alpha s^{\prime}\right) / s^{\prime} n}^{*} \subset A_{l\left(n-\alpha s^{\prime}\right) / s^{\prime} n}^{*} .
$$

Since $0<\alpha s^{\prime}<n, 1<p / s^{\prime}<n / \alpha s^{\prime}$, and $1 /\left(l / s^{\prime}\right)=1 /\left(p / s^{\prime}\right)-\alpha s^{\prime} / n$, using (2.2) again, we see that $(2.4)$ is equivalent to $\left(u^{s^{\prime}}, v^{s^{\prime}}\right) \in A^{*}\left(p / s^{\prime}, l / s^{\prime}\right)$.

Since $q / l<1$, by $(2.3)$ and (ii), we have

$$
\left(u^{q}, v^{q}\right)=\left(\left[u^{l}\right]^{q / l},\left[v^{l}\right]^{q / l}\right) \in A_{q\left(n-\alpha s^{\prime}\right) / s^{\prime} n}^{*} .
$$

Noting that $0<\alpha s^{\prime}<n, 1<t / s^{\prime}<n / \alpha s^{\prime}$, and $1 /\left(q / s^{\prime}\right)=1 /\left(t / s^{\prime}\right)-\alpha s^{\prime} / n$, we see that $(2.5)$ is equivalent to $\left(u^{s^{\prime}}, v^{s^{\prime}}\right) \in A^{*}\left(t / s^{\prime}, q / s^{\prime}\right)$ by (2.2). Thus we finish the proof of the conclusion (a).

Lemma 2.2. Suppose that $m \geq 1$ and $D^{\gamma} A \in L_{\mathrm{loc}}\left(\mathbb{R}^{n}\right)$ for $|\gamma|=m-1$. Then for a.e. $x, y \in$ $\mathbb{R}^{n}$,

$$
\left|R_{m}(A ; x, y)\right| \leq C|x-y|^{m-1} \sum_{|\gamma|=m-1}\left(\left(D^{\gamma} A\right)^{*}(x)+\left(D^{\gamma} A\right)^{*}(y)\right),
$$

where $f^{*}(x)$ denotes the Hardy-Littlewood maximal function of $f(x)$.

Proof. Obviously, $\left|R_{1}(A ; x, y)\right| \leq A^{*}(x)+A^{*}(y)$ for $m=1$. When $m>1$, we write

$$
R_{m}(A ; x, y)=R_{m-1}(A ; x, y)-\sum_{|y|=m-1} D^{\gamma} A(y)(x-y)^{\gamma} .
$$

Hence,

$$
\frac{\left|R_{m}(A ; x, y)\right|}{|x-y|^{m-1}} \leq \frac{\left|R_{m-1}(A ; x, y)\right|}{|x-y|^{m-1}}+C \sum_{|y|=m-1}\left|D^{\gamma} A(y)\right| .
$$

Thus by (2.8), if we can show that

$$
\frac{\left|R_{m-1}(A ; x, y)\right|}{|x-y|^{m-1}} \leq C \sum_{|y|=m-1}\left(\left(D^{\gamma} A\right)^{*}(x)+\left(D^{\gamma} A\right)^{*}(y)\right),
$$

then we get (2.6). 
Now we give the proof of (2.9). We take $\phi \in C_{0}^{\infty}\left(\mathbb{R}^{n}\right)$ with $0 \leq \phi(x) \leq 1$ such that $\phi(x)=1$ if $|x| \leq 1, \phi(x)=0$ if $|x| \geq 2$. Let $|x-y|=\varepsilon / 2$ and $\phi_{\varepsilon}(x)=\phi(x / \varepsilon)$. For $F \in$ $C_{0}^{1}\left(\mathbb{R}^{n}\right)$, by [10] we have $F(y)=c_{n} \int_{\mathbb{R}^{n}}\left\langle\nabla_{\xi} F,(y-\xi) /|y-\xi|^{n}\right\rangle d \xi$, where $\nabla_{\xi} F=\left(D_{1} F\right.$, $\left.D_{2} F, \ldots, D_{n} F\right)$ and $D_{j} F=\partial F / \partial \xi_{j}$. Moreover, $\langle\cdot, \cdot\rangle$ is the notation of the inner product. Hence,

$$
\begin{aligned}
\frac{\left|R_{m-1}(A ; x, y)\right|}{|x-y|^{m-1}}= & \frac{\left|R_{m-1}(A ; x, y) \phi_{\varepsilon}(x-y)\right|}{|x-y|^{m-1}} \\
= & C \frac{1}{|x-y|^{m-1}}\left|\int_{\mathbb{R}^{n}}\left\langle\nabla_{\xi}\left(R_{m-1}(A ; x, \xi) \phi_{\varepsilon}(x-\xi)\right), \frac{y-\xi}{|y-\xi|^{n}}\right\rangle d \xi\right| \\
\leq & C\left(\frac{1}{|x-y|^{m-1}}\left|\int_{\mathbb{R}^{n}}\left\langle\left(\nabla_{\xi} R_{m-1}(A ; x, \xi)\right) \phi_{\varepsilon}(x-\xi), \frac{y-\xi}{|y-\xi|^{n}}\right\rangle d \xi\right|\right. \\
& \left.+\frac{1}{|x-y|^{m-1}}\left|\int_{\mathbb{R}^{n}}\left\langle R_{m-1}(A ; x, \xi)\left(\nabla_{\xi} \phi_{\varepsilon}(x-\xi)\right), \frac{y-\xi}{|y-\xi|^{n}}\right\rangle d \xi\right|\right) \\
:= & I+I I .
\end{aligned}
$$

Because $\left\langle\nabla_{\xi}\left(R_{m-1}(A ; x, \xi)\right),(y-\xi)\right\rangle=\sum_{|\gamma|=m-1} D^{\gamma} A(\xi) \sum_{|j|=1}\left((x-\xi)^{\gamma-j}(y-\xi)^{j} /\right.$ $(m-j) !)$, we have

$$
\begin{aligned}
& \quad\left|\left\langle\left(\nabla_{\xi} R_{m-1}(A ; x, \xi)\right) \phi_{\varepsilon}(x-\xi),(y-\xi)\right\rangle\right| \\
& \quad \leq C \chi_{\{|x-\xi| \leq 2 \varepsilon\}}(\xi) \sum_{|\gamma|=m-1}\left|D^{\gamma} A(\xi)\right||x-\xi|^{m-2}|y-\xi| .
\end{aligned}
$$

Hence for $I$, we get

$$
\begin{aligned}
I & \leq \frac{C}{|x-y|^{m-1}} \sum_{|\gamma|=m-1} \int_{|x-\xi| \leq 2 \varepsilon} \frac{\left|D^{\gamma} A(\xi)\right||x-\xi|^{m-2}}{|y-\xi|^{n-1}} d \xi \\
& \leq C \sum_{|\gamma|=m-1} \frac{1}{\varepsilon} \int_{|y-\xi| \leq 3 \varepsilon} \frac{\left|D^{\gamma} A(\xi)\right|}{|y-\xi|^{n-1}} d \xi \leq C \sum_{|\gamma|=m-1}\left(D^{\gamma} A\right)^{*}(y) .
\end{aligned}
$$

To obtain the estimate of $I I$, we need to use an equality in [2],

$$
R_{m-1}(A ; x, \xi)=\sum_{|\gamma|=m-1} \frac{m-1}{\gamma !}(x-\xi)^{\prime \gamma} \int_{0}^{|x-\xi|} \rho^{m-2} D^{\gamma} A\left(x-\rho(x-\xi)^{\prime}\right) d \rho,
$$

where $y^{\prime}=y /|y| \in S^{n-1}$. Since for $j=1,2, \ldots, n$,

$$
D_{j}\left(\phi_{\varepsilon}(x-\xi)\right)=-\frac{1}{\varepsilon}\left(D_{j} \phi\right)_{\varepsilon}(x-\xi), \quad \operatorname{supp}\left\{\left(D_{j} \phi\right)_{\varepsilon}(x-\xi)\right\} \subset\{\xi: \varepsilon \leq|x-\xi| \leq 2 \varepsilon\}
$$


1840 Two-weight estimate for fractional integral operators

we have $|y-\xi| \sim|x-\xi|$ and

$$
\begin{aligned}
I I & \leq \sum_{|\gamma|=m-1} \frac{C}{\varepsilon^{m}} \int_{\varepsilon \leq|x-\xi| \leq 2 \varepsilon} \frac{(x-\xi)^{\prime \gamma}}{|x-\xi|^{n-1}} \int_{0}^{|x-\xi|} \rho^{m-2}\left|D^{\gamma} A\left(x-\rho(x-\xi)^{\prime}\right)\right| d \rho d \xi \\
& \leq C \sum_{|\gamma|=m-1} \frac{1}{\varepsilon^{m}} \int_{\varepsilon}^{2 \varepsilon} \int_{0}^{t} \rho^{m-1} \int_{S^{n-1}}\left|D^{\gamma} A\left(x-\rho u^{\prime}\right)\right| d u^{\prime} d \rho d t \\
& \leq C \sum_{|\gamma|=m-1} \frac{1}{\varepsilon^{m}} \int_{\mathcal{\varepsilon}}^{2 \varepsilon} \int_{|u| \leq t}\left|D^{\gamma} A(x-u)\right||u|^{m-n-1} d u d t \\
& \leq C \sum_{|\gamma|=m-1}\left(D^{\gamma} A\right)^{*}(x) \frac{1}{\mathcal{E}^{m}} \int_{\mathcal{\varepsilon}}^{2 \varepsilon} \sum_{j \geq 0}\left(2^{-j} t\right)^{m-1} d t \leq \sum_{|\gamma|=m-1}\left(D^{\gamma} A\right)^{*}(x) .
\end{aligned}
$$

Thus (2.9) follows from (2.12) and (2.15).

\section{Proof of Theorem 1.5}

Now we turn to the proof of Theorem 1.5. For $m \geq 1$, by (2.6), we get

$$
\begin{aligned}
\left|T_{\Omega, \alpha}^{A} f(x)\right| & \leq C \int_{\mathbb{R}^{n}} \sum_{|\gamma|=m-1}\left(\left(D^{\gamma} A\right)^{*}(x)+\left(D^{\gamma} A\right)^{*}(y)\right) \frac{|\Omega(x-y)|}{|x-y|^{n-\alpha}}|f(y)| d y \\
& =C \sum_{|\gamma|=m-1}\left[\left(D^{\gamma} A\right)^{*}(x) \cdot T_{|\Omega|, \alpha}(|f|)(x)+T_{|\Omega|, \alpha}\left(\left(D^{\gamma} A\right)^{*} \cdot|f|\right)(x)\right] .
\end{aligned}
$$

Thus,

$$
\begin{aligned}
&\left(\int_{\mathbb{R}^{n}}\left|T_{\Omega, \alpha}^{A} f(x) u(x)\right|^{q} d x\right)^{1 / q} \\
& \leq C \sum_{|\gamma|=m-1}\left\{\left(\int_{\mathbb{R}^{n}}\left[\left(D^{\gamma} A\right)^{*}(x) T_{|\Omega|, \alpha}(|f|) u(x)\right]^{q} d x\right)^{1 / q}\right. \\
&\left.+\left(\int_{\mathbb{R}^{n}}\left[T_{|\Omega|, \alpha}\left(\left(D^{\gamma} A\right)^{*}|f|\right)(x) u(x)\right]^{q} d x\right)^{1 / q}\right\}:=C \sum_{|\gamma|=m-1}(I+I I) .
\end{aligned}
$$

If $r=\infty$, then $t=p, l=q$. From (3.2) and $L^{\infty}$ boundedness of the Hardy-Littlewood maximal operator, it is easy to see that neither $I$ nor $I I$ is larger than

$$
\left\|\left(D^{\gamma} A\right)^{*}\right\|_{L^{\infty}}\left(\int_{\mathbb{R}^{n}}\left[T_{|\Omega|, \alpha}(|f|)(x) u(x)\right]^{q} d x\right)^{1 / q} .
$$

By Theorem 1.6, we know that when $r=\infty$, the conclusion of Theorem 1.5 holds. 
As for the case $1<r<\infty$, we first consider the condition (a). Since $1 / l=1 / q-1 / r$, we have $1 /(r / q)+1 /(l / q)=1$. Using Hölder's inequality, we get

$$
\begin{aligned}
I & =\left(\int_{\mathbb{R}^{n}}\left[\left(D^{\gamma} A\right)^{*}(x) T_{|\Omega|, \alpha}(|f|)(x) u(x)\right]^{q} d x\right)^{1 / q} \\
& \leq\left(\int_{\mathbb{R}^{n}}\left(D^{\gamma} A\right)^{*}(x)^{q \cdot r / q} d x\right)^{(1 / q) \cdot(q / r)}\left(\int_{\mathbb{R}^{n}}\left[T_{|\Omega|, \alpha}(|f|)(x) u(x)\right]^{q \cdot l / q} d x\right)^{(1 / q) \cdot(q / l)} \\
& =\left\|\left(D^{\gamma} A\right)^{*}\right\|_{L^{r}}\left\|T_{|\Omega|, \alpha}(|f|)\right\|_{L^{l}\left(u^{l}\right) .}
\end{aligned}
$$

By $1 / l=1 / p-\alpha / n, s^{\prime}<t<p$, and the conclusions (a) and (b) of Lemma 2.1, we have $\left(u^{s^{\prime}}, v^{s^{\prime}}\right) \in A^{*}\left(p / s^{\prime}, l / s^{\prime}\right)$ and $u^{s^{\prime}}, v^{s^{\prime}} \in A\left(p / s^{\prime}, l / s^{\prime}\right)$. Applying Theorem 1.6 for $(s, p, l)$ and $(u, v)$ and the $L^{r}(r>1)$ boundedness of Hardy-Littlewood maximal operator, we have

$$
I \leq C\left\|D^{\gamma} A\right\|_{L^{r}}\left(\int_{\mathbb{R}^{n}}|f(x) v(x)|^{p} d x\right)^{1 / p} .
$$

Now we estimate $I I$. Since $1>1 / t>1 / p$, so $1<t<p<n / \alpha$ and $1 / q=1 / t-\alpha / n$. By $s^{\prime}<t$, using the conclusions (a) and (b) of Lemma 2.1 again, we have $\left(u^{s^{\prime}}, v^{s^{\prime}}\right) \in A^{*}\left(t / s^{\prime}, q / s^{\prime}\right)$ and $u^{s^{\prime}}, v^{s^{\prime}} \in A\left(t / s^{\prime}, q / s^{\prime}\right)$. Hence by the weighted $\left(L^{t}, L^{q}\right)$ boundedness of $T_{\Omega, \alpha}$ with different weights (Theorem 1.6), the $L^{r}(r>1)$ boundedness of Hardy-Littlewood maximal operator and using Hölder's inequality for $r / t$ and $p / t$, we get

$$
\begin{aligned}
I I & =\left(\int_{\mathbb{R}^{n}}\left[T_{|\Omega|, \alpha}\left(\left(D^{\gamma} A\right)^{*}|f|\right)(x) u(x)\right]^{q} d x\right)^{1 / q} \\
& \leq C\left(\int_{\mathbb{R}^{n}}\left[\left(D^{\gamma} A\right)^{*}(x)|f(x)| v(x)\right]^{t} d x\right)^{1 / t} \leq C\left\|D^{\gamma} A\right\|_{L^{r}}\left(\int_{\mathbb{R}^{n}}|f(x) v(x)|^{p} d x\right)^{1 / p} .
\end{aligned}
$$

From (3.2)-(3.6), we prove that $T_{\Omega, \alpha}^{A}$ is a bounded operator from $L^{p}\left(v^{p}\right)$ to $L^{q}\left(u^{q}\right)$ when the condition (a) of Theorem 1.5 is satisfied.

Applying the conclusions (c), (d) of Lemma 2.1 and Theorem 1.6 under the condition (b), respectively, and by the same method as above, we may prove that when the condition (b) of Theorem 1.5 is satisfied, the operator $T_{\Omega, \alpha}^{A}$ is also bounded from $L^{p}\left(v^{p}\right)$ to $L^{q}\left(u^{q}\right)$. Here we omit the details.

Remark 3.1. As a corollary of Theorem 1.5, below we will show that the same conclusions hold also for the multilinear fractional maximal operator $M_{\Omega, \alpha}^{A}$ (see (1.6) for its definition). Using the idea of proving [5, Lemma 2], we can obtain the following pointwise relation between $M_{\Omega, \alpha}^{A}$ and $\bar{T}_{|\Omega|, \alpha}^{A}$.

Lemma 3.2. Let $0<\alpha<n, \Omega \in L^{1}\left(S^{n-1}\right)$. Then

$$
M_{\Omega, \alpha}^{A} f(x) \leq \bar{T}_{|\Omega|, \alpha}^{A}(|f|)(x) \quad \text { for } x \in \mathbb{R}^{n},
$$

where

$$
\bar{T}_{|\Omega|, \alpha}^{A}(|f|)(x)=\int_{\mathbb{R}^{n}} \frac{\left|R_{m}(A, x, y)\right|}{|x-y|^{n-\alpha+m-1}}|\Omega(x-y)||f(y)| d y .
$$




\section{Two-weight estimate for fractional integral operators}

From the process proving Theorem 1.5, it is easy to see that the conclusions of Theorem 1.5 hold still for $\bar{T}_{|\Omega|, \alpha}^{A}$. Combining this with Lemma 3.2, we can easily obtain the weighted $\left(L^{p}, L^{q}\right)$ boundedness of $M_{\Omega, \alpha}^{A}$ for different wights.

Remark 3.3. When $D^{\gamma} A \in \operatorname{BMO}\left(\mathbb{R}^{n}\right)$, the weighted boundedness of $T_{\Omega, \alpha}^{A}$ with the weight pair $(u, v)$ will be given in the following paper.

\section{Acknowledgments}

The author is grateful to the excellent referee for detailed critical comments and valuable suggestions. The research was supported by National Natural Science Foundation of China (Grant 10271016).

\section{References}

[1] B. Bajšanski and R. Coifman, On singular integrals, Singular Integrals (Proc. Sympos. Pure Math. Chicago, Ill., 1966), vol. 10, American Mathematical Society, Rhode Island, 1967, pp. $1-17$.

[2] J. Cohen, A sharp estimate for a multilinear singular integral in $\mathbb{R}^{n}$, Indiana Univ. Math. J. 30 (1981), no. 5, 693-702.

[3] J. Cohen and J. A. Gosselin, On multilinear singular integrals on $\mathbb{R}^{n}$, Studia Math. 72 (1982), no. 3, 199-223.

[4] _ A BMO estimate for multilinear singular integrals, Illinois J. Math. 30 (1986), no. 3, $445-464$.

[5] Y. Ding, Weak type bounds for a class of rough operators with power weights, Proc. Amer. Math. Soc. 125 (1997), no. 10, 2939-2942.

[6] Y. Ding and C.-C. Lin, Two-weight norm inequalities for the rough fractional integrals, Int. J. Math. Math. Sci. 25 (2001), no. 8, 517-524.

[7] Y. Ding and S. Lu, Weighted norm inequalities for fractional integral operators with rough kernel, Canad. J. Math. 50 (1998), no. 1, 29-39.

[8] J. García-Cuerva and J. L. Rubio de Francia, Weighted Norm Inequalities and Related Topics, North-Holland Mathematics Studies, vol. 116, North-Holland, Amsterdam, 1985.

[9] B. Muckenhoupt and R. L. Wheeden, Weighted norm inequalities for singular and fractional integrals, Trans. Amer. Math. Soc. 161 (1971), 249-258.

[10] E. M. Stein, Singular Integrals and Differentiability Properties of Functions, Princeton Mathematical Series, no. 30, Princeton University Press, New Jersey, 1970.

Yong Ding: Department of Mathematics, Beijing Normal University, Beijing 100875, China

E-mail address: dingy@bnu.edu.cn 


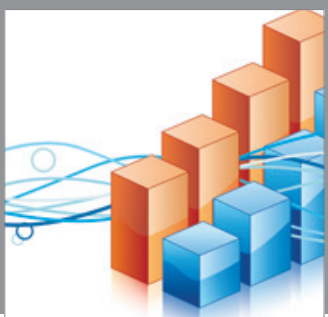

Advances in

Operations Research

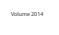

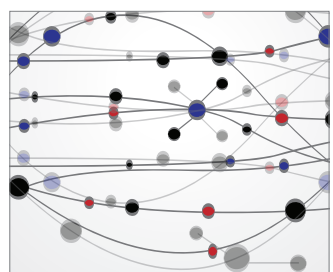

\section{The Scientific} World Journal
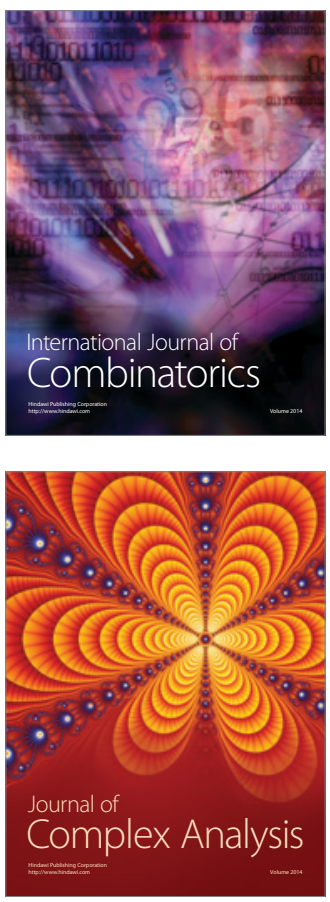

International Journal of

Mathematics and

Mathematical

Sciences
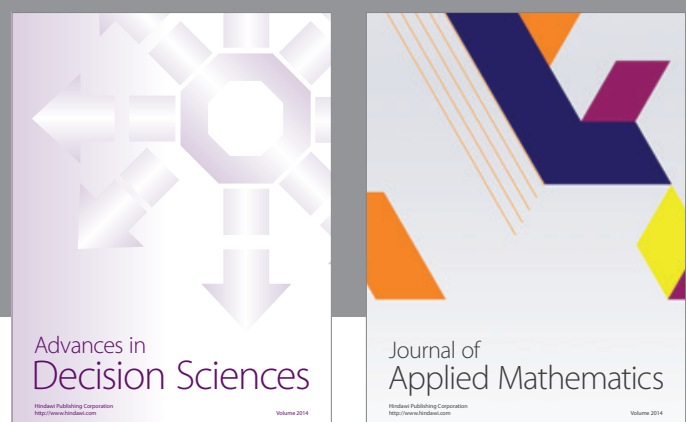

Journal of

Applied Mathematics
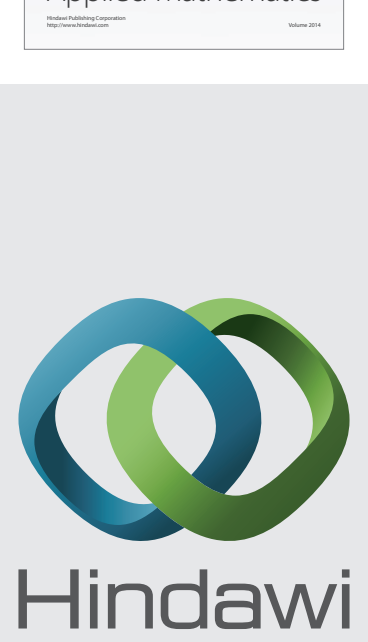

Submit your manuscripts at http://www.hindawi.com
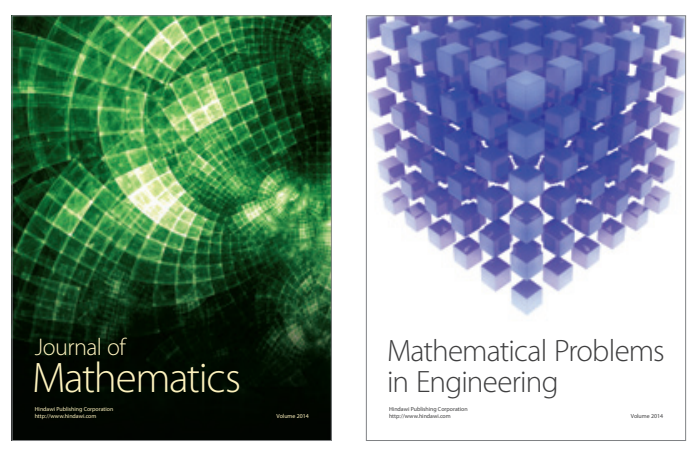

Mathematical Problems in Engineering
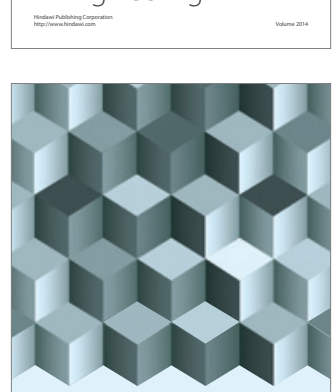

Journal of

Function Spaces
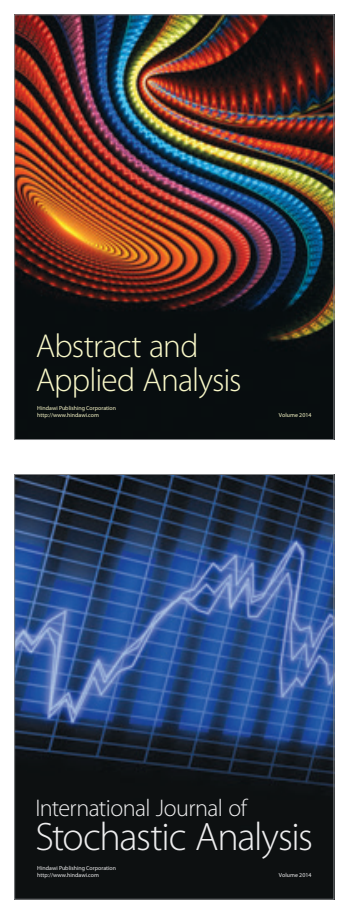

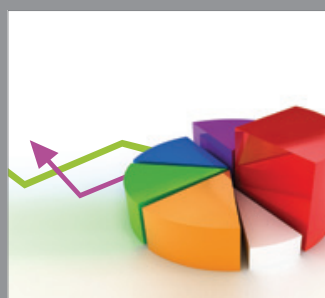

ournal of

Probability and Statistics

Promensencen
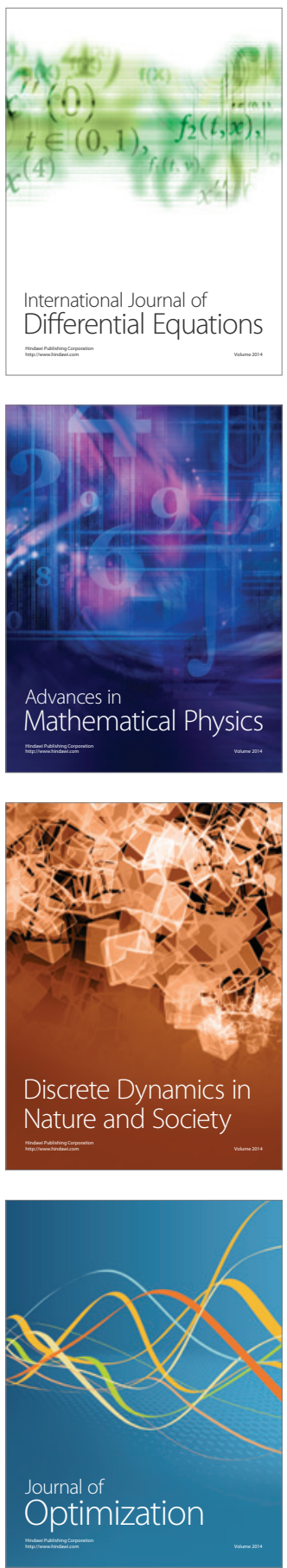